Check for updates

Cite this: RSC Adv., 2018, 8, 2851

\title{
Atmospheric-pressure-plasma-jet processed carbon nanotube (CNT)-reduced graphene oxide (rGO) nanocomposites for gel-electrolyte supercapacitors
}

\author{
Fei-Hong Kuok, ${ }^{a}$ Hung-Hua Chien, ${ }^{a}$ Chia-Chun Lee, ${ }^{a}$ Yu-Chuan Hao, ${ }^{b}$ Ing-Song Yu, \\ Cheng-Che Hsu, ${ }^{* c}$ I-Chun Cheng (DD) ${ }^{* d}$ and Jian-Zhang Chen (DD *a
}

This study evaluates DC-pulse nitrogen atmospheric-pressure-plasma-jet processed carbon nanotube (CNT)-reduced graphene oxide ( $\mathrm{rGO}$ ) nanocomposites for gel-electrolyte supercapacitor applications. $\mathrm{X}$-ray photoelectron spectroscopy (XPS) indicates decreased oxygen content (mainly, C-O bonding content) after nitrogen APPJ processing owing to the oxidation and vaporization of ethyl cellulose. Nitrogen APPJ processing introduces nitrogen doping and improves the hydrophilicity of the CNT-rGO nanocomposites. Raman analysis indicates that nitrogen APPJ processing introduces defects and/or surface functional groups on the nanocomposites. The processed CNT-rGO nanocomposites on carbon cloth are applied to the electrodes of $\mathrm{H}_{2} \mathrm{SO}_{4}$-polyvinyl alcohol (PVA) gel-electrolyte supercapacitors. The best achieved specific (areal) capacitance is $93.1 \mathrm{~F} \mathrm{~g}^{-1}\left(9.1 \mathrm{mF} \mathrm{cm}{ }^{-2}\right)$ with $15 \mathrm{~s}$ APPJ-processed CNT-rGO nanocomposite electrodes, as evaluated by cyclic voltammetry under a potential scan rate of $2 \mathrm{mV} \mathrm{s}^{-1}$. The addition of rGOs in CNTs in the nanoporous electrodes improves the supercapacitor performance.

Received 3rd November 2017 Accepted 5th January 2018

DOI: $10.1039 / c 7 r a 12108 c$

rsc.li/rsc-advances

\section{Introduction}

Atmospheric-pressure plasma (APP) technology can be operated at atmospheric pressure without using vacuum chambers and pumps that are costly and require routine maintenance. Therefore, it is considered a cost-effective technology. Typical APP technology includes dielectric barrier discharge (DBD), corona discharge, and atmospheric-pressure plasma jet (APPJ). ${ }^{1-3}$ Thermal plasma jets are operated in local thermal equilibrium (LTE), that is, the equilibrium between the electron temperature and the heavy particle temperature. On the other hand, non-LTE plasma jets or cold plasma jets have a heavy particle temperature ranged of 300-1000 K, which is lower than the electron temperature by at least one order of magnitude. APPJ is operated in open ambient at moderate gas temperatures. This high-reactivity plasma chemistry has attracted much research attention and has resulted in various practical applications. It produces charge

${ }^{a}$ Graduate Institute of Applied Mechanics, National Taiwan University, Taipei City 10617, Taiwan.E-mail: jchen@ntu.edu.tw

${ }^{b}$ Department of Materials Science and Engineering, National Dong Hwa University, Hualien 97401, Taiwan

${ }^{c}$ Department of Chemical Engineering, National Taiwan University, Taipei City 10617, Taiwan.E-mail: chsu@ntu.edu.tw

${ }^{d}$ Graduate Institute of Photonics and Optoelectronics, Department of Electrical Engineering, National Taiwan University, Taipei City 10617, Taiwan. E-mail: iccheng@ntu.edu.tw particles, neutral metastable species, radicals, and ultraviolet (UV) radiation. ${ }^{4}$ Owing to these features, APP technology has been applied to food and agriculture processing, ${ }^{5-7}$ biomedicine ${ }^{8,9}$ and materials processing. ${ }^{\mathbf{1 0 - 1 5}}$

Batteries usually possess high energy density but low power density; on the other hand, supercapacitor is the other way around. There have been some attempts to design cells that compromise or keep both advantages. ${ }^{\mathbf{1 6}}$ Supercapacitors are passive electrochemical energy storage devices that have high power density and long cyclic lifetime. Electrode materials are essential for high-performance supercapacitors. Many studies have focused on developing new electrode materials with economic processing techniques. ${ }^{17-21}$ Two main charge storage mechanisms are typically involved in supercapacitors: faradaic pseudocapacitance and/or electrical double layer capacitance (EDLC). Pseudocapacitance results from reversible redox reactions on electrode material surfaces, and it usually occurs in metal oxides and conductive polymers. ${ }^{17,22-29}$ EDLC originates from the charge accumulation at electrolyte/electrode, and it usually occurs in carbonaceous electrode materials. ${ }^{30-33}$ CNTs has been used for supercapacitors with EDLC due to its high electrical conductivity and superior mechanical elasticity of the tube-shaped structures. ${ }^{34,35}$ It is recommended that CNTs can be used as a great support for other active materials. Graphene is also introduced to electrochemical devices because of its outstanding electrical and thermal properties. By interlayering 
CNTs and graphenes, the supercapacitor performance can be greatly improved. ${ }^{36}$ The high specific surface area and chemical stability of graphene can enhance the supercapacitor performance, while CNTs provide a tubular network for graphene. ${ }^{37}$ Graphene and CNT composites also have been applied as transparent conductors. ${ }^{38}$

DC-pulse nitrogen APPJs operated at a substrate temperature of $400-800{ }^{\circ} \mathrm{C}$, which is self-sustained by the plasma jet. They show high reactivity with carbonaceous materials such as reduced graphene oxide (rGO) nanoflakes, carbon nanotubes (CNTs), fibers of carbon cloth, and graphite felts. ${ }^{\mathbf{1 0 3 9 - 4 5}}$ Strong -CN violet emissions are identified in plasma during the early stage of APPJ processing of carbonaceous materials. This validates the occurrence of vigorous interactions between nitrogen reactive plasma species and carbon-based materials. In addition, the rapid etching of polyimide has been realized using non-LTE APPJs; reactive oxygen species are considered effective for this rapid etching process. ${ }^{\mathbf{4 6}, 47}$ Ultrafast mask-free etching of CNT micro-supercapacitors has also been achieved; in this process, ozone generated by the APPJ serves as the main oxidants. ${ }^{48}$ In this study, we investigate CNT-rGO nanocomposite gel-electrolyte supercapacitors processed by DC-pulse nitrogen APPJs. Nitrogen APPJs quickly oxidized/vaporized ethyl cellulose binders to form nanoporous CNT-rGO nanocomposites. Owing to the synergetic effects of the reactive nitrogen plasma species and heat, the optimal processing time is merely $15 \mathrm{~s}$. In comparison to our previous study with CNT supercapacitors, ${ }^{39}$ the addition of rGO into CNT electrodes improves supercapacitor performance.

\section{Experimental details}

\section{Preparation of CNT-rGO pastes for screen printing}

0.05 g of CNTs (purity: $>98 \%$, diameter: $5-20 \mathrm{~nm}$, length: $>1 \mu \mathrm{m}$, Golden Innovation Business Co., Ltd.), $0.05 \mathrm{~g}$ of rGOs (purity 99\%, thickness < $5 \mathrm{~nm}$, sheet diameter: 0.1-5 $\mu \mathrm{m}$, Golden Innovation Business Co., Ltd.), $1.75 \mathrm{~g}$ of $10 \mathrm{wt} \%$ ethyl cellulose (5-15 mPa s, \#46070, Fluka) ethanoic solution, $2.25 \mathrm{~g}$ of $10 \mathrm{wt} \%$ ethyl cellulose (30-50 mPa s, \#46080, Fluka) ethanoic solution, $3.245 \mathrm{~g}$ of terpineol (anhydrous, \#86480, Fluka), and $1.5 \mathrm{~g}$ of ethanol were mixed and stirred at room temperature for $24 \mathrm{~h}$ using a magnetic stirrer. Then, $4 \mathrm{ml}$ of this mixture was extracted and condensed at $55{ }^{\circ} \mathrm{C}$ for $7 \mathrm{~min}$ using a rotovap.

\section{Fabrication of CNT-rGO nanocomposite electrodes on carbon cloth}

CNT-rGO pastes were screen-printed onto carbon cloth, following which a DC-pulse nitrogen APPJ was applied to process the printed pastes for $0 / 5 / 15 / 30 \mathrm{~s}$. The APPJ operations have been described in our previous study. ${ }^{49}$ Fig. 1 shows the substrate temperature evolution as monitored using a K-type thermocouple equipped with a data acquisition device (USB-6221, National Instruments) connected to a computer. After the APPJ was turned on, the substrate temperature increased rapidly to $\sim 700{ }^{\circ} \mathrm{C}$ and then gradually reached a steady state. The temperature decreased dramatically after the APPJ was turned off. During APPJ

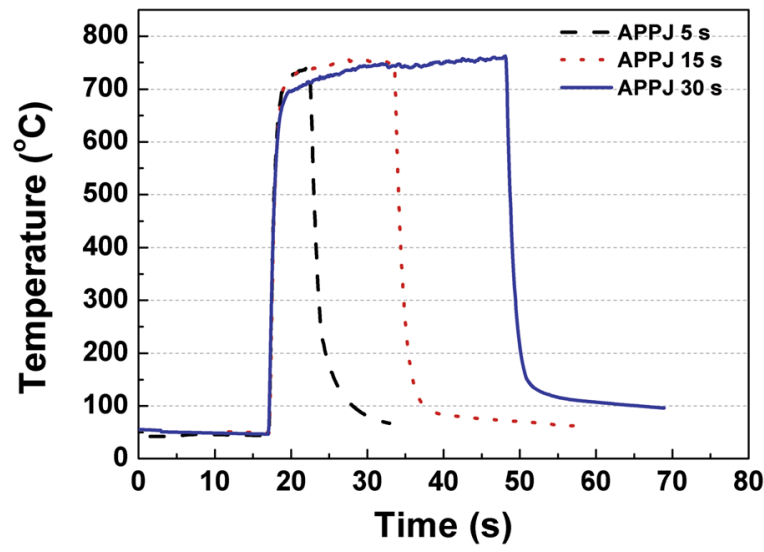

Fig. 1 Substrate temperature evolution under APPJ operation.

processing, the optical emission spectra (OES) were monitored using a spectrometer (Ocean Optics, Flame).

\section{Materials characterization of CNT-rGO nanocomposites on carbon cloth}

Scanning electron microscopy (SEM, Nova NanoSEM 230, FEI) was used to inspect the CNT-rGO nanocomposites on carbon cloth. X-ray photoelectron spectroscopy (XPS, VGS Thermo Scientific) was used for surface chemical analyses. An electron probe X-ray microanalyzer (EPMA, JEOL JXA-8200) was used for elemental chemical composition analyses. Raman spectroscopy (Renishaw 1000B) was used to inspect the degrees of disordering, defect, and impurity of the CNT-rGO nanocomposites. A goniometer (Model 100SB, Sindatek Instruments Co., Ltd.) was used to measure the water contact angles of the CNT-rGO nanocomposites before/after APPJ processing.

\section{Fabrication of gel-electrolyte supercapacitors}

APPJ-processed CNT-rGO nanocomposites on carbon cloth were used as the electrodes of a symmetric supercapacitor. A gel-electrolyte was prepared by mixing $15 \mathrm{ml}$ of $1 \mathrm{M} \mathrm{H}_{2} \mathrm{SO}_{4}$ and $1.5 \mathrm{~g}$ of polyvinyl alcohol (PVA) at $80{ }^{\circ} \mathrm{C}$ for $4 \mathrm{~h}$. The gelelectrolyte was spread onto two pieces of APPJ-processed CNT-rGO nanocomposites on carbon cloth and naturally dried for $24 \mathrm{~h}$. Two pieces of gel-coated electrodes were then pressed together on the gel sides to form a sandwich-type supercapacitor.

\section{Characterization of supercapacitors}

Cyclic voltammetry (CV) and CV cycling stability tests with a potential scan window of $0-0.8 \mathrm{~V}$ were performed using an electrochemical workstation (Zahner Zennium) by the twoelectrode method (CNT-coated carbon cloth electrodes).

\section{Results and discussion}

Fig. 2(a) shows a photograph of the APPJ during APPJ processing. After the APPJ was turned on, its color was blue-purple; then, the color quickly turned pink after a short duration. 

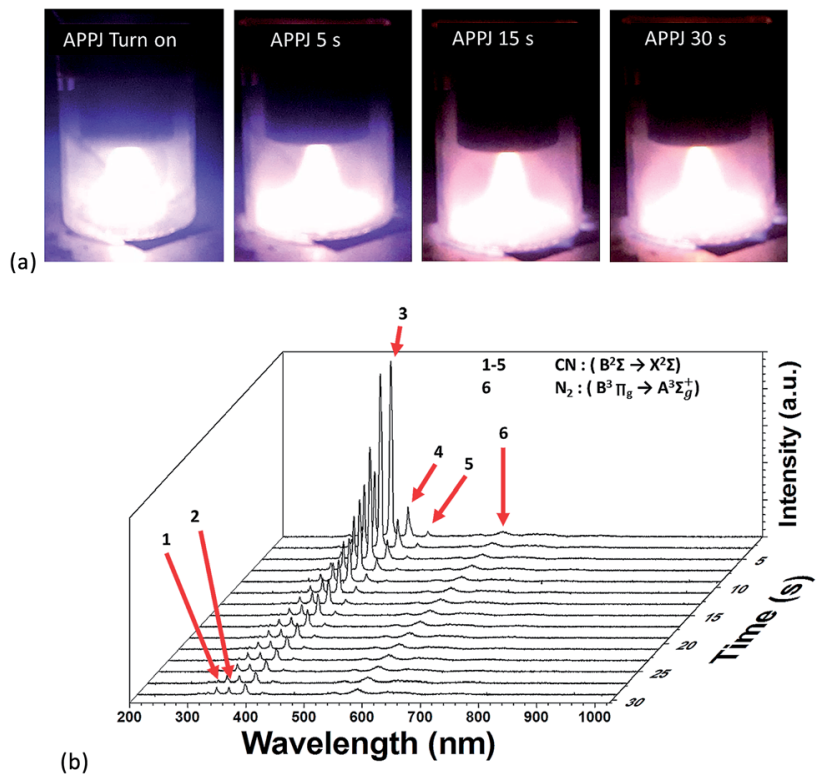

Fig. 2 (a) Photographs of APPJs during APPJ processing on screenprinted CNT-rGO nanocomposites. (b) Evolution of OES during APPJ processing on screen-printed CNT-rGO nanocomposites.

Fig. 2(b) shows the evolution of OES during APPJ processing. The intensity of $\mathrm{CN}$ emissions $(359,388,418$, and $453 \mathrm{~nm}$ ) increased rapidly and then decreased with the APPJ processing time. After a certain period, some remnant $\mathrm{N}_{2} 2^{\text {nd }}$ positive emissions that overlapped with the $\mathrm{CN}$ emissions were present. The other remaining emissions were persistent $\mathrm{N}_{2} 1^{\text {st }}$ positive emission $\left(\mathrm{B}^{3} \Pi_{\mathrm{g}} \rightarrow \mathrm{A}^{3} \Sigma_{\mathrm{u}}^{+}\right)$at wavelengths longer than $530 \mathrm{~nm}$. This suggested the violent reaction of carbonaceous materials with the reactive nitrogen species in plasma. Molecules containing $-\mathrm{CN}$ bonds were excited and then relaxed to emit characteristic optical spectra.

Fig. 3 shows SEM images of as-deposited CNT-rGO nanocomposites (without APPJ-calcination) and CNT-rGO nanocomposites processed by APPJs for various durations. The largediameter $(\sim 10 \mu \mathrm{m})$ cylinder-shaped materials are carbon cloth
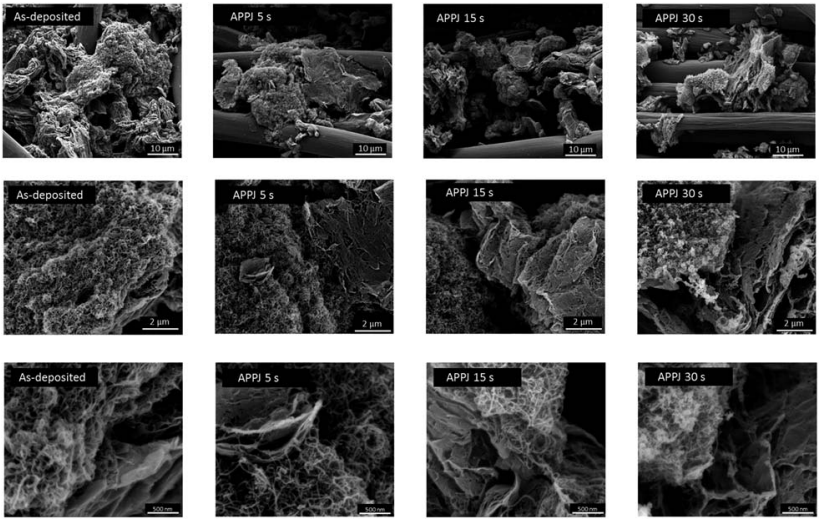

Fig. 3 SEM images of as-deposited CNT-rGO nanocomposites (without APPJ-calcination) and CNT-rGO nanocomposites processed by APPJ for various durations.

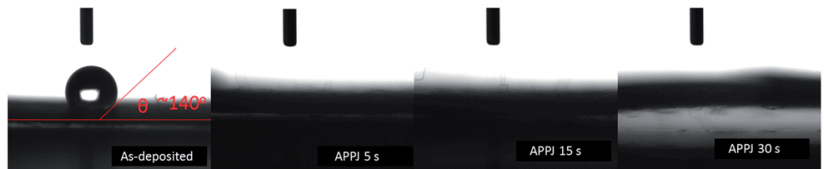

Fig. 4 Water contact angle of screen-printed CNT-rGO nanocomposites before/after APPJ processing.

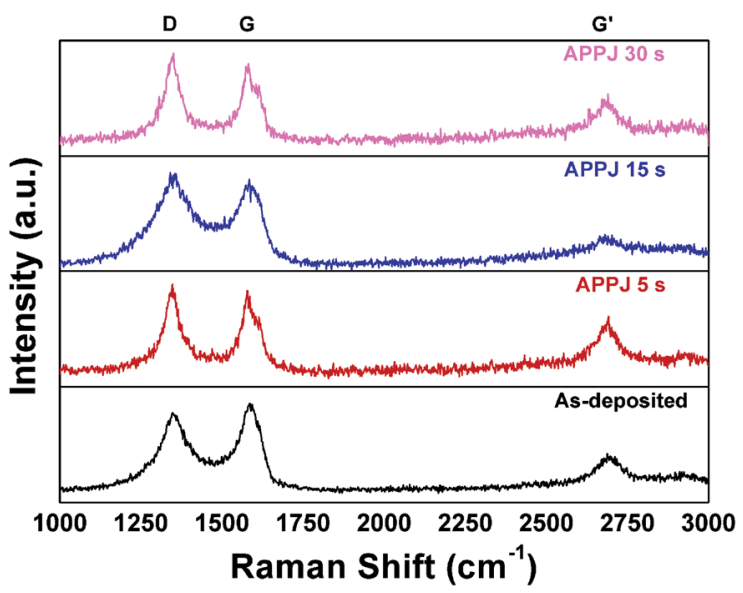

Fig. 5 Raman spectra of CNT-rGO nanocomposites before/after APPJ processing.

fibers. CNTs and rGO nanoflakes form nanoporous composite structure.

Fig. 4 shows the water contact angle measurement results of the screen-printed CNT-rGO nanocomposites before/after APPJ processing. Without APPJ processing, the screen-printed CNTrGO shows a water contact angle as large as $\sim 140^{\circ}$. After APPJ processing for $5 \mathrm{~s}$, the screen-printed CNT-rGO and carbon cloth fibers became highly hydrophilic, and testing water droplets completely penetrated the nanopores.

Fig. 5 shows the Raman spectra of the CNT-rGO nanocomposites processed by APPJ for various durations. The Dband $\left(\sim 1350 \mathrm{~cm}^{-1}\right)$, G-band $\left(\sim 1580 \mathrm{~cm}^{-1}\right)$, and $\mathrm{G}^{\prime}$-band $\left(\sim 2700 \mathrm{~cm}^{-1}\right)$ are identified in the spectra. ${ }^{50-53}$ The stretching of the $\mathrm{C}-\mathrm{C}$ bond in graphitic materials contributes to the G-band featuring an $\mathrm{sp}^{2}$ carbon system. ${ }^{51}$ The D-band typically indicates carbonaceous impurities with $\mathrm{sp}^{3}$ bonding or broken $\mathrm{sp}^{2}$ bonds. ${ }^{50,51}$ The G'-band is a second-order two-phonon process corresponding to long-range ordering in a sample. ${ }^{50,51}$ The intensity ratio of the D-band and G-band $\left(I_{\mathrm{D}} / I_{\mathrm{G}}\right)$ is frequently used for purity or defect assessment. ${ }^{50,53} I_{\mathrm{D}} / I_{\mathrm{G}}$ was 0.92 before APPJ processing, and this ratio increased to 1.07 after APPJ processing. This suggests that reactive species in nitrogen APPJs interact with the CNT-rGO nanocomposites and introduce some defects or surface functional groups to the nanocomposites.

Fig. 6 shows a wide scan of the CNT-rGO nanocomposites before/after APPJ processing. Typical $\mathrm{C} 1 \mathrm{~s}$ and O1s binding energy peaks are present. These are typical elemental compositions of CNT, rGO, and binders (ethyl cellulose, terpineol, and 


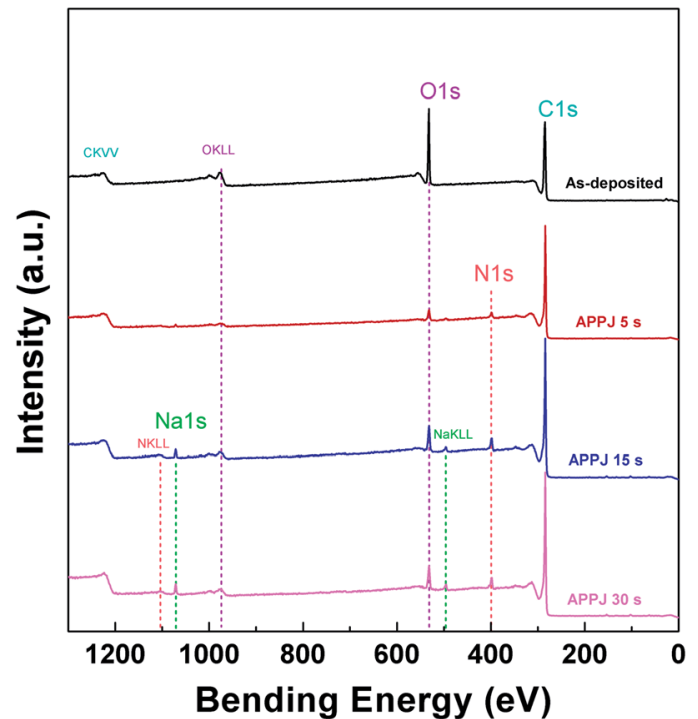

Fig. 6 XPS wide scan of CNT-rGO nanocomposites on carbon cloth.

ethanol). After APPJ processing, N1s and Na1s signals started to appear. Table 1 shows the elemental composition analyzed from XPS and EPMA. EPMA also shows Na signals from all samples including those without APPJ processing. This Na contamination could result from the APPJ, environment, or personal handling. The appearance of the N1s signal indicates nitrogen doping on CNT-rGO nanocomposites and carbon cloth fibers introduced by nitrogen APPJ processing. The overall oxygen content also significantly decreased after APPJ processing, indicating the oxidation and vaporization of terpineol and ethyl cellulose after APPJ processing. As the APPJ processing time increased, the oxygen content recovered slightly, possibly owing to the oxidation process caused by the reaction among reactive plasma species in nitrogen APPJ, environmental oxygen, and the materials.

Apparent shape changes of XPS C1s and N1s fine scan spectra were observed for CNT-rGO nanocomposites with/ without APPJ processing. Fig. 7 shows the XPS C1s fine scan spectra. The as-deposited CNT-rGO (without APPJ processing) shows a strong $\mathrm{C}-\mathrm{O}$ peak at $\sim 286.1 \mathrm{eV}$. This originates mainly from the ethyl cellulose. ${ }^{54}$ The $\mathrm{C}-\mathrm{O}$ peak decreased dramatically after APPJ processing, indicating the removal of a significant amount of ethyl cellulose by the nitrogen APPJ. The C-C content was mainly contributed by CNTs, rGOs, and the carbon cloth fibers. Fig. 8 shows the XPS N1s fine scan spectra of the CNT-
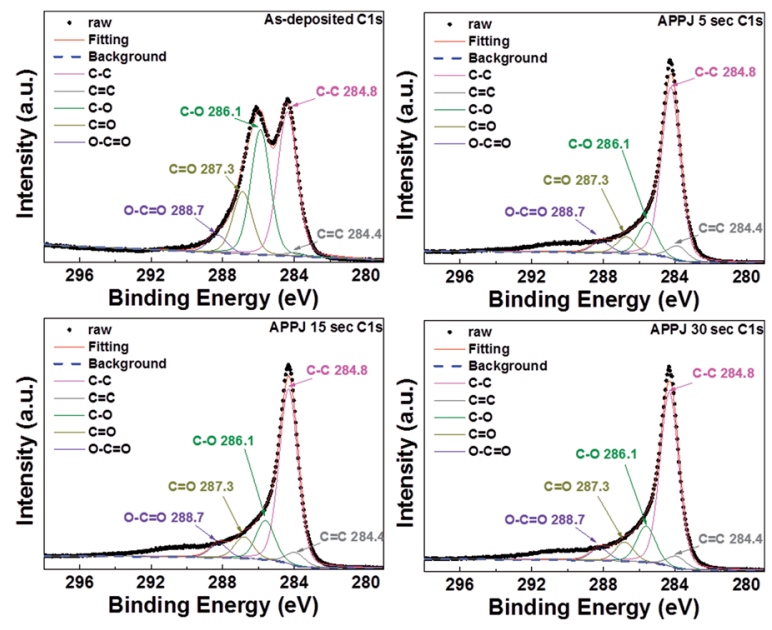

Fig. 7 XPS C1s fine scan spectra of CNT-rGO nanocomposites before/after APPJ processing.

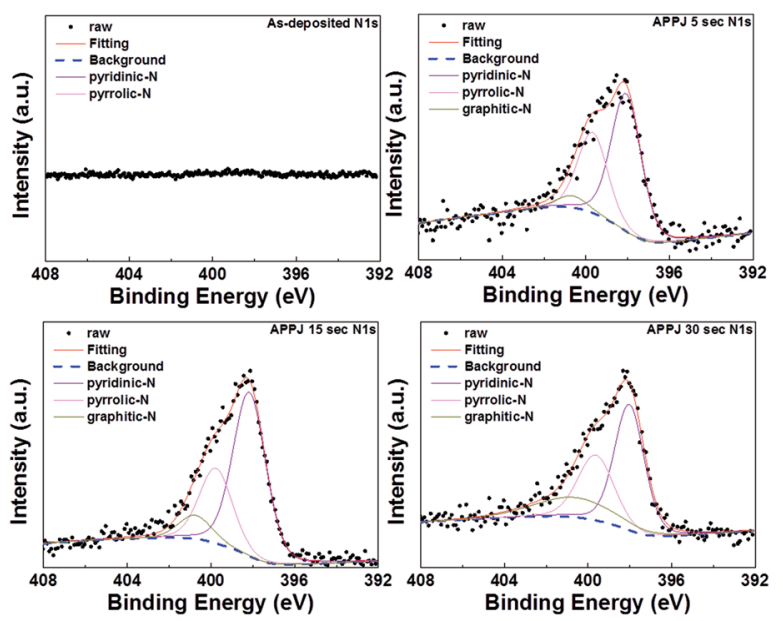

Fig. 8 XPS N1s fine scan spectra of CNT-rGO nanocomposites before/after APPJ processing.

rGO nanocomposite before/after APPJ processing. Without APPJ processing, no N1s signal was observed. After APPJ processing, the N1s signal was noted. This suggests that nitrogen APPJ resulted in the nitrogen doping on CNTs, rGOs, and/or carbon cloth fibers. Nitrogen doping in the carbon-based material was considered to benefit the capacitive performance of the supercapacitor because of the introduction of pseudocapacitance. ${ }^{20,55,56}$ However, pseudocapacitance effect is not noted in

Table 1 C, N, O, and Na relative compositions as measured using XPS and EPMA

\begin{tabular}{|c|c|c|c|c|c|c|c|c|}
\hline \multirow[b]{2}{*}{ at $\%$} & \multicolumn{4}{|l|}{ XPS } & \multicolumn{4}{|l|}{ EPMA } \\
\hline & $\mathrm{C}$ & $\mathrm{N}$ & $\mathrm{O}$ & $\mathrm{Na}$ & $\mathrm{C}$ & $\mathrm{N}$ & $\mathrm{O}$ & $\mathrm{Na}$ \\
\hline As-deposited & 74.70 & $\sim 0$ & 25.30 & $\sim 0$ & 99.11 & $\sim 0$ & 0.556 & 0.3305 \\
\hline APPJ $5 \mathrm{~s}$ & 89.82 & 4.227 & 5.461 & 0.487 & 95.06 & 4.394 & 0.174 & 0.3640 \\
\hline APPJ $15 \mathrm{~s}$ & 83.87 & 5.996 & 9.119 & 1.012 & 96.28 & 3.307 & 0.157 & 0.2485 \\
\hline APPJ $30 \mathrm{~s}$ & 85.05 & 5.236 & 8.392 & 1.313 & 95.75 & 3.853 & 0.145 & 0.2466 \\
\hline
\end{tabular}



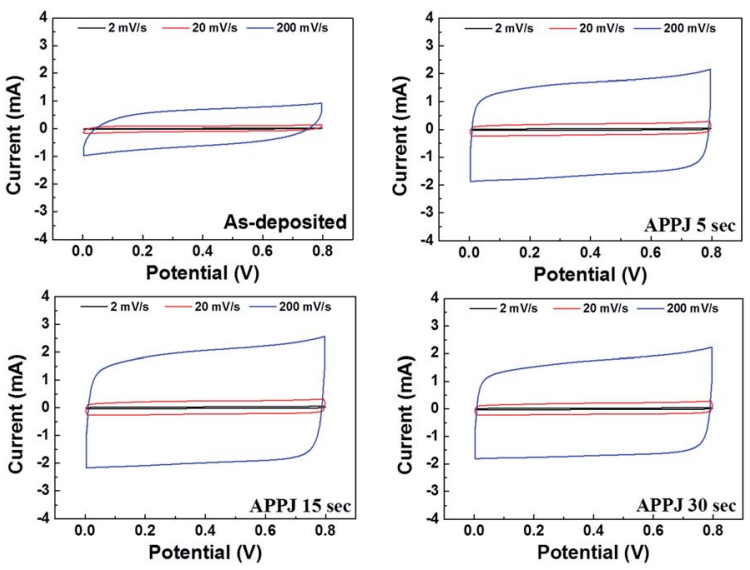

Fig. 9 CV curves of supercapacitors with electrodes processed by APPJ for various durations.

our samples; the redox peaks are not observed in the CV curves of Fig. 9. The introduction of nitrogen doping in carbon materials could also improve hydrophilicity. ${ }^{55}$ The improvement of hydrophilicity could facilitate the contact between the electrolyte and the nanoporous electrode materials, leading to improved capacitance.

Fig. 9 shows the CV results obtained by characterizing the supercapacitors. The specific capacitance can be calculated based on the following equation. ${ }^{17}$

$$
C_{\mathrm{sp}}=\frac{1}{m v\left(V_{\mathrm{b}}-V_{\mathrm{a}}\right)} \int_{V_{\mathrm{a}}}^{V_{\mathrm{b}}} I(V) \mathrm{d} V
$$

where $C_{\mathrm{sp}}$ is the specific capacitance; $m$, the mass of the printed material; $v$, the potential scan rate; $V_{\mathrm{b}}-V_{\mathrm{a}}$, the potential scan window; and $I(V)$, the response current at voltage $V$ under $\mathrm{CV}$ operation. The areal capacitance can be obtained by replacing $m$ with the electrode apparent area $A$. Table 2 lists the calculated capacitance values based on Fig. 9. APPJ processing significantly improved the performance of supercapacitors. The capacitance value reached a maximum value of $93.1 \mathrm{~F} \mathrm{~g}^{-1}$ $\left(9.10 \mathrm{mF} \mathrm{cm}^{-2}\right)$ with $15 \mathrm{~s}$ APPJ processing. APPJ processing can remove the nonconductive ethyl cellulose binders, thereby improving the capacitance. Furthermore, the improved wettability may facilitate the penetration of the gel-electrolyte into nanopores of the nanocomposites and increase the contact area between the electrolyte and the electrode materials, thereby improving the performance of supercapacitors. In comparison with APPJ-processed CNT supercapacitors, ${ }^{40}$ the addition of rGOs apparently improved the capacitance values, possibly

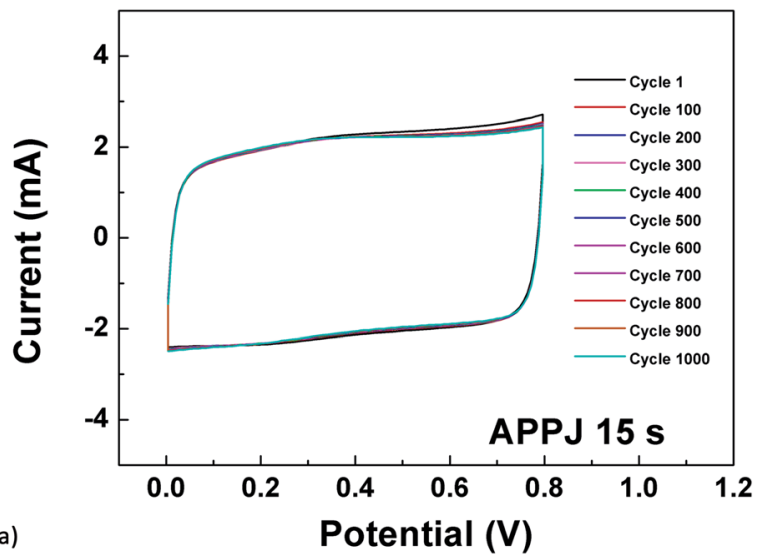

(a)

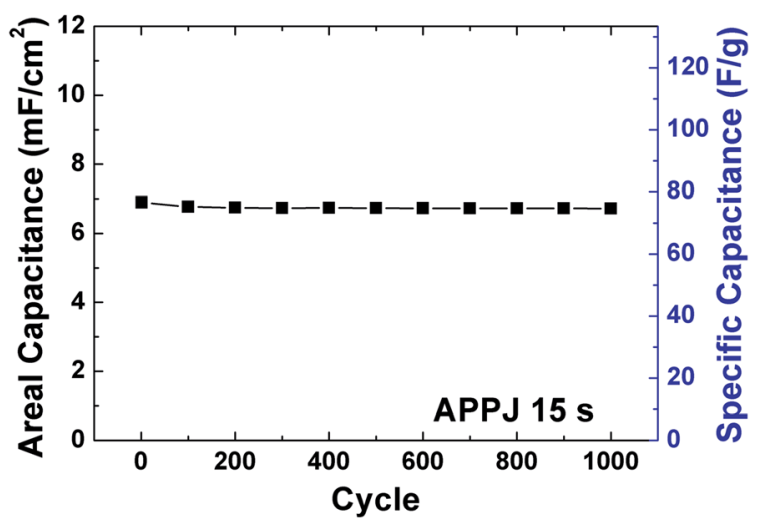

Fig. 10 (a) CV cycling stability test evaluated under a potential scan rate of $200 \mathrm{mV} \mathrm{s}^{-1}$. (b) Capacitance versus cycle number.

owing to the larger surface-to-volume ratio of rGO nanoflakes compared with that of CNTs.

The stability of the best achieved supercapacitor with $15 \mathrm{~s}$ APPJ processing was evaluated by cycling CV tests under a potential scan rate of $200 \mathrm{mV} \mathrm{s}^{-1}$. Fig. 10(a) shows the cycling CV curves spaced by 100 cycles. The CV curves almost overlap except for the curves of cycle 1 and cycle 100. Fig. 10(b) shows the capacitance value versus cycle number. The capacitance retention rate is $97.46 \%$ after $1000 \mathrm{CV}$ cycling tests. The stability is comparable to that of APPJ-processed CNT supercapacitors whose capacitance retention rate is $94.7 \%$ after $1000 \mathrm{CV}$ cycles.

\section{Summary}

We evaluate CNT-rGO nanocomposite gel-electrolyte supercapacitors processed by DC-pulse nitrogen APPJs. XPS analyses

Table 2 Capacitance values evaluated by CV under potential scan rates of 2, 20, and $200 \mathrm{mV} \mathrm{s}^{-1}$

\begin{tabular}{|c|c|c|c|c|c|c|c|c|}
\hline $\begin{array}{l}\text { Scan rate } \\
\left(\mathrm{mV} \mathrm{s}^{-1}\right)\end{array}$ & \multicolumn{4}{|c|}{ Specific capacitance $\left(\mathrm{F} \mathrm{g}^{-1}\right)$} & \multicolumn{4}{|c|}{ Areal capacitance $\left(\mathrm{mF} \mathrm{cm}^{-2}\right)$} \\
\hline 20 & 35.9 & 70.0 & 77.6 & 69.7 & 3.23 & 6.43 & 7.58 & 6.46 \\
\hline 200 & 21.6 & 58.0 & 65.4 & 59.3 & 1.94 & 5.33 & 6.40 & 5.49 \\
\hline
\end{tabular}


indicate that APPJ processing results in the removal of ethyl cellulose and introduction of nitrogen doping. The hydrophilicity is also significantly improved by the nitrogen APPJ processing; this facilitates the penetration of the gel-electrolyte into nanopores to improve the contact between the electrolyte and the electrode materials, thereby improving the supercapacitor performance. Raman analyses suggest the introduction of defects and/or surface functional groups on CNT-rGO nanocomposites and carbon cloth fibers. The best achieved specific (areal) capacitance is $93.1 \mathrm{~F} \mathrm{~g}^{-1}\left(9.1 \mathrm{mF} \mathrm{cm}^{-2}\right)$ with $15 \mathrm{~s}$ APPJprocessed CNT-rGO nanocomposite electrodes, as evaluated by $\mathrm{CV}$ under a potential scan rate of $2 \mathrm{mV} \mathrm{s}^{-1}$. Mixing rGOs into CNTs in the nanoporous electrodes improves the supercapacitor performance.

\section{Conflicts of interest}

There are no conflicts to declare.

\section{Acknowledgements}

The authors gratefully acknowledge the funding support from the Ministry of Science and Technology of Taiwan under grant nos. MOST 105-2221-E-002-047-MY3 and MOST 106-2221-E-002193-MY2. A cleanroom facility was provided by the NanoElectro-Mechanical-Systems (NEMS) Research Center at National Taiwan University. We would also like to thank $\mathrm{Mr}$ Chung-Yuan Kao and Ms. Yuan-Tzu Lee of the Instrumentation Center at National Taiwan University for their assistance with the EPMA and SEM operations, respectively.

\section{Notes and references}

1 A. Schutze, J. Y. Jeong, S. E. Babayan, J. Park, G. S. Selwyn and R. F. Hicks, IEEE Trans. Plasma Sci., 1998, 26, 1685-1694.

2 M. Laroussi and T. Akan, Plasma Processes Polym., 2007, 4, 777-788.

3 C. Tendero, C. Tixier, P. Tristant, J. Desmaison and P. Leprince, Spectrochim. Acta, Part B, 2006, 61, 2-30.

4 J. Winter, R. Brandenburg and K. D. Weltmann, Plasma Sources Sci. Technol., 2015, 24.

5 B. Surowsky, O. Schluter and D. Knorr, Food Eng. Rev., 2015, 7, 82-108.

6 L. K. Randeniya and G. J. J. B. de Groot, Plasma Processes Polym., 2015, 12, 608-623.

7 Z. C. Zong, R. W. Zhou, D. P. Liu, Y. Song and J. H. Niu, Plasma Processes Polym., 2016, 13, 402-409.

8 G. Y. Park, S. J. Park, M. Y. Choi, I. G. Koo, J. H. Byun, J. W. Hong, J. Y. Sim, G. J. Collins and J. K. Lee, Plasma Sources Sci. Technol., 2012, 21.

9 L. F. Gaunt, C. B. Beggs and G. E. Georghiou, IEEE Trans. Plasma Sci., 2006, 34, 1257-1269.

10 J. Z. Chen, W. Y. Liao, W. Y. Hsieh, C. C. Hsu and Y. S. Chen, J. Power Sources, 2015, 274, 894-898.

11 T. Homola, P. Dzik, M. Vesely, J. Kelar, M. Cernak and M. Weiter, ACS Appl. Mater. Interfaces, 2016, 8, 33562-33571.
12 L. Bonova, A. Zahoranova, D. Kovacik, M. Zahoran, M. Micusik and M. Cernak, Appl. Surf. Sci., 2015, 331, 79-86. 13 O. V. Penkov, M. Khadem, W. S. Lim and D. E. Kim, J. Coat. Technol. Res., 2015, 12, 225-235.

14 C. Y. Chou, H. M. Chang, H. W. Liu, Y. J. Yang, C. C. Hsu, I. C. Cheng and J. Z. Chen, RSC Adv., 2015, 5, 45662-45667. 15 T. J. Wu, C. Y. Chou, C. M. Hsu, C. C. Hsu, J. Z. Chen and I. C. Cheng, RSC Adv., 2015, 5, 99654-99657.

16 H. Jiang, H. X. Zhang, Y. Fu, S. J. Guo, Y. J. Hu, L. Zhang, Y. Liu, H. L. Liu and C. Z. Li, ACS Nano, 2016, 10, 1648-1654.

17 G. S. Gund, D. P. Dubal, N. R. Chodankar, J. Y. Cho, P. Gomez-Romero, C. Park and C. D. Lokhande, Sci. Rep., 2015, 5, 12454.

18 C. H. Xu, P. Y. Shen, Y. F. Chiu, P. W. Yeh, C. C. Chen, L. C. Chen, C. C. Hsu, I. C. Cheng and J. Z. Chen, J. Alloys Compd., 2016, 676, 469-473.

19 C. Y. Liao, F. H. Kuok, C. W. Chen, C. C. Hsu and J. Z. Chen, Journal of Energy Storage, 2017, 11, 237-241.

20 Z. Gao, X. Liu, J. Chang, D. Wu, F. Xu, L. Zhang, W. Du and K. Jiang, J. Power Sources, 2017, 337, 25-35.

21 Q. Chen, X. M. Li, X. B. Zang, Y. C. Cao, Y. J. He, P. X. Li, K. L. Wang, J. Q. Wei, D. H. Wu and H. W. Zhu, RSC Adv., 2014, 4, 36253-36256.

22 M. Moussa, M. F. El-kady, Z. Zhao, P. Majewski and J. Ma, Nanotechnology, 2016, 27, 442001.

23 C. Yang, L. L. Zhang, N. T. Hu, Z. Yang, H. Wei, Z. C. J. Xu, Y. Y. Wang and Y. F. Zhang, Appl. Surf. Sci., 2016, 379, 206-212.

24 N. L. Chen, Y. P. Ren, P. P. Kong, L. Tan, H. X. Feng and Y. C. Luo, Appl. Surf. Sci., 2017, 392, 71-79.

25 Z. X. Song, Y. J. Zhang, W. Liu, S. Zhang, G. C. Liu, H. Y. Chen and J. S. Qiu, Electrochim. Acta, 2013, 112, 120-126.

26 M. Aadil, W. Shaheen, M. F. Warsi, M. Shahid, M. A. Khan, Z. Ali, S. Haider and I. Shakir, J. Alloys Compd., 2016, 689, 648-654.

27 Y. Zhang, L. Zu, H. Q. Lian, Z. K. Hu, Y. H. Jiang, Y. Liu, X. D. Wang and X. G. Cui, J. Alloys Compd., 2017, 694, 136144.

28 R. M. Kore and B. J. Lokhande, J. Alloys Compd., 2017, 725, 129-138.

29 Q. Q. Qin, X. D. Du, C. X. Xu, S. G. Huang, W. J. Wang, Y. Zhang, J. Yan, J. Q. Liu and Y. C. Wu, J. Electrochem. Soc., 2017, 164, A1952-A1957.

30 L. L. Zhang and X. Zhao, Chem. Soc. Rev., 2009, 38, 25202531.

31 H. M. Jeong, J. W. Lee, W. H. Shin, Y. J. Choi, H. J. Shin, J. K. Kang and J. W. Choi, Nano Lett., 2011, 11, 2472-2477.

32 Y. W. Zhu, S. Murali, M. D. Stoller, K. J. Ganesh, W. W. Cai, P. J. Ferreira, A. Pirkle, R. M. Wallace, K. A. Cychosz, M. Thommes, D. Su, E. A. Stach and R. S. Ruoff, Science, 2011, 332, 1537-1541.

33 L. Wang, L. Sun, C. Tian, T. Tan, G. Mu, H. Zhang and H. Fu, RSC Adv., 2012, 2, 8359-8367.

34 V. V. Obreja, Phys. E, 2008, 40, 2596-2605.

35 R. H. Baughman, A. A. Zakhidov and W. A. De Heer, Science, 2002, 297, 787-792. 
36 H. Jiang, P. S. Lee and C. Li, Energy Environ. Sci., 2013, 6, 4153.

37 D. Yu and L. Dai, J. Phys. Chem. Lett., 2009, 1, 467-470.

38 V. C. Tung, L.-M. Chen, M. J. Allen, J. K. Wassei, K. Nelson, R. B. Kaner and Y. Yang, Nano Lett., 2009, 9, 1949-1955.

39 F. H. Kuok, K. Y. Kan, I. S. Yu, C. W. Chen, C. C. Hsu, I. C. Cheng and J. Z. Chen, Appl. Surf. Sci., 2017, 425, 321328.

40 F. H. Kuok, C. Y. Liao, T. H. Wan, P. W. Yeh, I. C. Cheng and J. Z. Chen, J. Alloys Compd., 2017, 692, 558-562.

41 C.-H. Yang, F.-H. Kuok, C.-Y. Liao, T.-H. Wan, C.-W. Chen, C.-C. Hsu, I.-C. Cheng and J.-Z. Chen, Mater. Res. Express, 2017, 4, 025504.

42 H. W. Liu, S. P. Liang, T. J. Wu, H. Chang, P. K. Kao, C. C. Hsu, J. Z. Chen, P. T. Chou and I. C. Cheng, ACS Appl. Mater. Interfaces, 2014, 6, 15105-15112.

43 S. H. Chang, J. S. Liou, J. L. Liu, Y. F. Chiu, C. H. Xu, B. Y. Chen and J. Z. Chen, J. Power Sources, 2016, 336, 99-106.

44 J. Z. Chen, C. Wang, C. C. Hsu and I. C. Cheng, Carbon, 2016, 98, 34-40.

45 T. H. Wan, Y. F. Chiu, C. W. Chen, C. C. Hsu, I. C. Cheng and J. Z. Chen, Coatings, 2016, 6, 44.
46 J. Y. Jeong, S. E. Babayan, V. J. Tu, J. Park, I. Henins, R. F. Hicks and G. S. Selwyn, Plasma Sources Sci. Technol., 1998, 7, 282-285.

47 J. Y. Jeong, S. E. Babayan, A. Schutze, V. J. Tu, J. Park, I. Henins, G. S. Selwyn and R. F. Hicks, J. Vac. Sci. Technol., A, 1999, 17, 2581-2585.

48 L. Liu, D. Ye, Y. Yu, L. Liu and Y. Wu, Carbon, 2017, 111, 121127.

49 C. H. Xu, Y. F. Chiu, P. W. Yeh and J. Z. Chen, Mater. Res. Express, 2016, 3, 085002.

50 R. A. DiLeo, B. J. Landi and R. P. Raffaelle, J. Appl. Phys., 2007, 101.

51 M. S. Dresselhaus, A. Jorio, M. Hofmann, G. Dresselhaus and R. Saito, Nano Lett., 2010, 10, 751-758.

52 D. K. Singh, P. K. Iyer and P. K. Giri, Diamond Relat. Mater., 2010, 19, 1281-1288.

53 L. Bokobza and J. Zhang, eXPRESS Polym. Lett., 2012, 6, 601608.

54 F. H. Kuok, Master degree thesis, National Taiwan University, 2017.

55 Z.-j. Jiang, Z. Jiang and W. Chen, J. Power Sources, 2014, 251, 55-65.

56 L. Sun, L. Wang, C. Tian, T. Tan, Y. Xie, K. Shi, M. Li and H. Fu, RSC Adv., 2012, 2, 4498-4506. 